\title{
Statistical Analysis of Ion Mobility Spectrometry. II. Adaptively Biased Methods and Shape Correlations
}

\author{
Florent Calvo, ${ }^{1,2}$ Fabien Chirot, ${ }^{1,3}$ Florian Albrieux, ${ }^{1,2,3}$ Jérôme Lemoine, ${ }^{1,3}$ \\ Yury O. Tsybin, ${ }^{4}$ Pascal Pernot, ${ }^{5,6}$ Philippe Dugourd ${ }^{1,2}$ \\ ${ }^{1}$ Université de Lyon, Université Lyon 1, Villeurbanne, 69622 France \\ ${ }^{2}$ CNRS, UMR 5579, LASIM, Villeurbanne, 69622 France \\ ${ }^{3}$ CNRS, UMR 5280, ISA, Villeurbanne, 69622 France \\ ${ }^{4}$ Biomolecular Mass Spectrometry Laboratory, Ecole Polytechnique Fédérale de Lausanne, 1015 Lausanne, Switzerland \\ ${ }^{5}$ Université Paris-Sud, Orsay, 91405 France \\ ${ }^{6}$ CNRS, UMR 8000, LCP, Orsay, 91405 France
}

\begin{abstract}
Following a recent effort [J. Am. Soc. Mass Spectrom. 23, 386-396 (2012)], we continue to explore computational methodologies for generating molecular conformations to support collisional cross sections suggested by ion mobility measurements. Here, adaptively biased molecular dynamics (ABMD) simulations are used to sample the configuration space and to achieve flat-histogram sampling along the reaction coordinates of the first two moments of the gyration tensor. The method is tested and compared with replica-exchange simulations on triplyprotonated bradykinin and on a larger 25-residue peptide. It is found to have a much higher efficiency for producing large sets of conformations in a broad range of diffusion cross-sections, whereas it does not compete with conventional replica-exchange molecular dynamics in locating the lowest-energy structure. Nevertheless, the broad sampling obtained from the ABMD method allows to quantitatively correlate the diffusion cross-section $\Omega$ with other geometric order parameters that have simpler interpretation. The strong correlations found between the diffusion cross-section and the radius of gyration, the surface area and the volume of the convex hull suggest an optimal template for accurately mimicking the variations of $\Omega$ in a broad range of conformations, using only geometrical information and doing so at a very moderate computational cost. The existence of such a correlation is confirmed on the much larger protein a-lactalbumin.
\end{abstract}

Key words: Ion mobility spectrometry, Structure determination, Molecular dynamics

\section{Introduction}

$I^{-}$ on mobility spectrometry (IMS) with mass-selected compounds has recently led to major advances in our understanding of the tertiary structure of peptides and proteins [1-3] and to the assembly and folding mechanisms of biomolecular complexes [4-8] in the gas phase. As is the

Correspondence to: Fabien Chirot; e-mail: fabien.chirot@univ-lyon1.fr case for spectroscopic analyses, the efficiency of this experimental technique for unraveling molecular conformations is bound to a successful comparison with calculated structures. For small systems, chemical intuition and trialand-error procedures can be sufficient to generate conformations that account for the measurements. However, due to the variety of intramolecular chemical interactions in terms of strength and range, the number of stable isomers in peptides increases very fast with the number of amino acids, 
and systematic computational approaches are required already for peptides with more than 10 residues. One major difficulty of theoretical chemical methods is their lack of energetic accuracy, even at the sophisticated levels of explicit electronic structure methods [9-11]. In addition, they are not practical for exploring the potential energy landscape. In this respect, biomolecular force fields, despite lacking chemical accuracy as well [11], remain inescapable. Current powerful algorithms for sampling energy landscapes include replica-exchange molecular dynamics (REMD) [12], which is particularly suited to statistical analyses using the weighted histograms method [13], and flat histogram strategies such as the Wang-Landau method [14, 15], integrated tempering [16], self-healing umbrella sampling [17], or metadynamics [18]. Sampling is not only necessary for locating the stable structures without a priori bias, it is also crucial for producing statistical sets of conformations that are relevant under the finite temperature of the experiment, and to account for specific thermal effects due to conformational broadening.

The second bottleneck of theoretical interpretations is the ability to calculate the measured properties for a given conformation. In the case of IMS, several schemes are available to determine the collision cross-section under realistic drift tube conditions [19-23]. The projection approximation (PA) of Bowers and coworkers [19] is probably the simplest, as atoms are approximated as hard spheres, neglecting the momentum transfer between the buffer gas and the analyte $[19,20]$. The effect of long-range potential can nevertheless be accounted for in this simple frame using the model implemented by Wyttenbach et al. [21]. This approach has been recently improved by treating the atoms as soft spheres and considering concavity corrections [23]. The exact hard-sphere scattering (EHSS) method [20] and the direct trajectory (TJ) method [22] of Jarrold and coworkers both take into account momentum transfer and are based on simulating trajectories of the impinging buffer gas atom. In principle, the trajectory approach is the most realistic and accurate, as it accounts for short- and long-range interactions, including polarization forces. Unfortunately, all these methods require solving computationally expensive numerical integrals, either to evaluate the area of a projected union of disks in the PA method, or to solve the multidimensional collision integral in both the EHSS and TJ methods, the latter being significantly more time-consuming since atom trajectories have to be calculated. In addition, the procedure has to be repeated for each new structure of the analyte, leading to a significant burden in case where chemical intuition fails to produce candidate conformations.

Although increasingly successful for interpreting ion mobility measurements $[4,5,8,24]$, conventional molecular dynamics simulations may also face difficulties in locating conformations compatible with experiments due to either intrinsic force field limitations, or insufficient sampling time [24]. One useful strategy to facilitate interpretation of IMS measurements consists in guiding the sampling toward the regions of experimental interest, using a dedicated order parameter that hopefully mimics the measured property. The collision cross section itself cannot be used actively in such MD simulations, because it is not an explicit function of the coordinates (no gradient is available), but the gyration radius turns out to be a reasonable approximation [24]. It should be stressed here that the biased MD approach does not aim at approximating the CCS by a geometrical quantity, but to take advantage of the relation between the CCS and the molecular structure in order to save computational time in the production of realistic candidate structures. Indeed, the existence of such a relation is at the basis of the use of IMS as a conformational probe. One straightforward biasing method uses a so-called umbrella potential, in which conformations with prescribed values of the order parameter are favored. However, locating conformations with prescribed values of the collision cross section was still a challenge with this approach, the parameters for the guiding potential having to be adjusted by trial and error [24].

The present work aims to extend this previous effort along two different directions, both in concern with the theoretical interpretation of IMS data. Instead of the tedious optimization of the umbrella parameters, systematic flathistogram methods are available to sample conformations uniformly over a range of order parameters [18, 25]. Here we use the adaptively biased molecular dynamics (ABMD) approach [26], which is an extension of the metadynamics method where the biasing potential is stored as polynomials on a grid, rather than a growing series of Gaussian functions in the original version [18]. The ABMD method is used in two dimensions, with the guiding order parameters given by the lowest moments of the gyration tensor. Whereas the first moment is equivalent to the square gyration radius, the second moment provides a measure of the deviation to sphericity, and is expected to capture more structural details. The broad sampling offered by the ABMD approach can be exploited to correlate the key quantity in IMS, namely the collision cross section, to other geometrical or energetic properties. Such correlations could be helpful in designing approximate representations of the collision cross-section that are both accurate and computationally inexpensive.

Following our previous work [24], we have chosen to test these ideas on selected peptides for which experimental IMS data are available. Triply protonated bradykinin was recently found by the Clemmer group [27, 28] to exhibit three distinct signatures in ion mobility spectrometry upon specific activation. In addition, we have studied a variant of a domain of the M2 trans-membrane protein from influenza virus A (M2TMP), which displays a gas phase bi-stable structure with a competition between helices and more compact structures [29]. These two peptides were modeled using the AMBER force field [28], which is a good compromise in terms of efficiency and accuracy. Although we do not seek here to interpret specific experiments for these two peptides, they are sufficiently large to provide suitable testing ground for the computational methodologies 
we wish to explore. In particular, and as will be shown below, they confirm the superiority of flat-histogram methods to sample broad sets of conformations, without requiring specific guiding potentials. Moreover, the statistical correlations found between the collision cross-section and various structural parameters suggest that it is indeed possible to approximate the former property as a direct, purely geometrical quantity. We have verified this conjecture in a broader size range, by performing additional simulations for a realistic protein containing about 2000 atoms, namely $\alpha$-lactalbumin. Our results for this system confirm the high degree of correlation with purely geometrical quantities, thus paving the way toward more efficient guiding strategies in simulations of large biomolecules for interpretation of IMS data.

In the next section, we briefly describe the main principle of the adaptively biased molecular dynamics method, and the definition of the order parameters based on the gyration tensor. Alternative simulations based on REMD are also discussed. The third section compares the relative performances of the two approaches for sampling the energy landscapes of the two selected peptides. The fourth section discusses the correlations between the collision cross section and various other physicochemical properties of the peptides, and it also includes some results for the much larger $\alpha$ lactalbumin protein, confirming the general findings obtained on the smaller peptides.

\section{Methods}

\section{Adaptively Biased Molecular Dynamics}

The standard replica-exchange method accelerates barrier crossing at low temperature by communicating the information from higher temperature trajectories [11, 29, 30], preserving the overall statistics of the canonical ensemble. In contrast, flat-histogram approaches [12-17,31] attempt to populate conformation space uniformly along one or several order parameters, typically by penalizing configurations increasingly as they are being visited. In doing so, the system evolves accordingly with biased statistics, and the canonical ensemble can be recovered afterwards by unbiasing. When the potential energy is chosen as the order parameter, the microcanonical density of states is obtained as an output of the penalty function, with the Wang-Landau method [14] being a typical example. In most cases, explicit order parameters chosen based on intuition guide the sampling, and the Landau free energy is eventually obtained from the bias. Here we use one of such methods as a scheme more systematic than umbrella sampling [32] to explore molecular conformations over a broad range of collision cross-sections. For simplicity we denote by $\mathbf{R}=\left\{\boldsymbol{r}_{i}\right\}$ the set of atomic positions of the system, and by $V(\mathbf{R})$ the potential energy at configuration $\mathbf{R}$.

The ABMD method [26] is an adaptation of metadynamics [18], in which a time-dependent biasing potential $W(t, \mathbf{R})$ is added to $V$ in such a way that the distribution along a reaction coordinate $\Lambda(\mathbf{R})$ will become asymptotically flat. In the original metadynamics method, at every time step $t$ the current configuration $\mathbf{R}(t)$ contributes to $W$ by a Gaussian function centered on $\mathbf{R}$ and with an adjustable width. The continuous addition of penalty functions make the evaluation of the biasing potential scale poorly at long times, and Gaussians are also particularly costly to compute away from their center. These two drawbacks are cured by the adaptively biased MD method, in which $W$ is described on a grid and the Gaussians are replaced by cubic B-splines with finite support. The full details of the method can be found in the paper by Babin and coworkers [26], but its main elements are now repeated. $W$ is first written as the sum of basis functions with time-dependent coefficients $a_{k}(t)$, each function acting on a single element of the grid [26]:

$$
W(t, \lambda)=\sum_{k \in \mathbb{Z}} \alpha_{k}(t) B\left(\frac{\lambda}{\Delta \lambda}-k\right)
$$

with $k \in \mathbb{Z}$ an integer, $\Delta \lambda$ a kernel width, and $B(x)$ a cubic Bspline defined piecewise as

$$
B(x)=\left\{\begin{array}{cc}
\frac{2}{3}+\frac{x^{2}(|x|-2)}{2} & \text { if }-1 \leq|x| \leq 1 \\
\frac{\left(2-|x|^{3}\right)}{6} & \text { if } 1 \leq|x| \leq 2 \\
0 & \text { otherwise }
\end{array}\right.
$$

The biasing function evolves in time according to a simple first-order Equation [26]:

$$
\frac{\partial W}{\partial t}=\frac{k_{\mathrm{B}} T}{\tau_{\mathrm{F}}} G(\lambda-\lambda(\mathbf{R}))
$$

where $G(x)$ is a biweight kernel defined by $G(x)=48\left(1-x^{2} / 4\right)^{2} / 41$ for $|x|<2$, and 0 otherwise, and $\tau_{\mathrm{F}}$ is a flooding time [26]. In the above equation, $k_{\mathrm{B}}$ and $T$ denote the Boltzmann constant and the temperature, respectively. Whereas the equations of motion are solved by the second-order velocity Verlet integrator, $W$ is updated by a simpler Euler scheme. The two control parameters of the method, $\Delta \lambda$ and $\tau_{\mathrm{F}}$, must be adjusted to optimize uniform sampling. The basic ABMD method can be extended in various ways, and in the present work we have used multiple walkers sharing a common biasing function $[26,33]$. In practice, a number $N_{W}$ of independent walkers are propagated in parallel, but contrary to parallel tempering they do not swap configurations, and instead all contribute to evolving the common biasing function. As done previously [34], we have also set up a regulation procedure in which the histograms are periodically monitored, and the replica located at the most populated place is deleted, whereas the replica located at the least populated place is duplicated. This replication/deletion scheme is only used during equilibration, but enhances convergence and helps the replicas to spread over the entire range of order parameter.

The ABMD method can also be straightforwardly adapted to multidimensional biasing over sets of coordinates $\lambda_{1}, \ldots, \lambda_{p}$, using higher order tensors for the coefficients 
$\alpha_{k_{1}}(t) \ldots \alpha_{k_{p}}(t)$ and products of B-splines in the relevant intervals. One concern in molecular dynamics simulations is the need for the gradient of the biasing potential, which requires the order parameters to be differentiable. This is unfortunately not the case for the collision cross-section, which also turns out to be computationally expensive, especially when calculated by the trajectory method.

\section{Order Parameters}

As an alternative to the collision cross-section, other order parameters are needed to guide sampling, hopefully leading to broad distributions in $\Omega$ as well. The squared gyration radius $R_{g}^{2}$ is of primary importance in the physics and chemistry of polymers, and it was used in our previous work together with umbrella sampling biasing [29]. It was then found to be an acceptable approximation to $\Omega$, although a significant effort was required in order to estimate the optimal value of $R_{g}^{2}$ meeting a target $\Omega$. As a natural extension to the gyration radius, we have considered higher moments of the gyration tensor for capturing additional structural details that are lacking in the first moment $R_{g}^{2}$. The gyration tensor $\mathbf{S}=\left(S_{\alpha \beta}\right)$ is defined from its six components as

$$
S_{\alpha \beta}=\frac{1}{N} \sum_{i} r_{i}^{\alpha} r_{i}^{\beta}
$$

where $N$ is the number of atoms in the system and $\alpha$ denotes one of the $x, y$, or $z$ components. Notice that we do not weigh the contribution of each atom by its mass, as we expect little difference in the results by doing so. The trace $\xi$ of $\mathbf{S}$ is equal to $R_{g}^{2}$, and we define the tensor $\mathbf{D}$ as $\mathbf{D}=\mathbf{S}-(\xi / 3) \mathbf{I}$, where $\mathbf{I}$ is the $3 \times 3$ identity matrix, so that $\mathbf{D}$ is traceless. The asphericity and prolateness parameters $A$ and $P$ are respectively defined from the successive powers of $\mathbf{D}$ as: [35]

$$
\begin{aligned}
& A=3 \frac{\operatorname{Tr}\left(\mathbf{D}^{2}\right)}{2 \xi^{2}} \\
& B=3 \frac{\operatorname{Tr}\left(\mathbf{D}^{3}\right)}{2 \xi^{3}}
\end{aligned}
$$

With those definitions, $A$ is always positive, vanishes for spherical symmetry and is always lower than 1 , whereas $-1 / 8 \leq P \leq 1$, oblate (prolate) shapes corresponding to negative (positive) values [35]. More importantly for use in biased dynamics, these parameters are all differentiable, even though the expressions for the gradient become increasingly cumbersome as higher moments of $\mathbf{D}$ are considered.

A multidimensional implementation of the ABMD method was carried out using the variables $\xi, A$, and $P$, and it was soon concluded that the additional consideration of the prolateness parameter was not significantly beneficial, as it entailed lower resolutions for each dimension of the histograms without affecting the quality of the sampling in the other two variables. All the results shown hereafter were thus obtained by biasing only on the two first moments $\xi$ and $A$ of the gyration tensor.

\section{Replica-Exchange Molecular Dynamics}

The efficiency of the ABMD method in producing conformations with a broad distribution of collision cross-sections is assessed by comparison with replica-exchange molecular dynamics simulations for the same peptides, using $N_{T}$ replicas with temperatures allocated according to a geometric progression in a fixed range $T_{\min }-T_{\max }$. Exchanges between configurations from adjacent replicas were attempted with a fixed time period of $\tau_{\mathrm{ex}}$. For both methods, a same number of configurations were saved for further structural analysis.

\section{Observables}

The conformational analysis was achieved based on several geometrical and physical properties. The collision cross section $\Omega$ was calculated using the direct trajectory method [22]. The shape parameters $\xi, A$, and $P$ were obtained from the successive moments of the gyration tensor. Additional information about the convexity of the molecular structure was inferred from the surface area $s$ and the volume $v$ of the convex hull of the atomic positions, calculated using the quickhull program [36]. It is important to stress here that, contrary to collision crosssections, the convex hull is not computationally expensive and scales favorably with the number of atoms. We also performed an inherent structure analysis, by systematically quenching each saved configuration into its closest stable minimum with energy $E_{\min }$. Local optimizations were carried out using the conjugate gradient algorithm.

\section{Computational Details}

Triply-protonated bradykinin (sequence RPPGFSPFR) and the G13L variant of a transmembrane domain of the M2 protein from influenza virus A (sequence SSDPLVVAASIILILHLILWILDRL) were modeled using the non-polarizable AMBER 99 force field [30]. For both peptides, protonation was assumed at the most basic sites, including arginine, histidine, and N-terminus. We also applied some of the computational methodology to the eightfold deprotonated $\alpha$ lactalbumin protein (PDB entry 1F6S), to which the disulfide bridges were removed in order to gain flexibility. This much larger molecule was also simulated with the same force field, in the ABMD framework.

The underlying engine of the ABMD and REMD methods is a classical velocity Verlet integrator solving the extended equations of motion with Nosé-Hoover thermostat chosen with mass $Q=(3 \mathrm{~N}-6) k_{\mathrm{B}} T / \Omega^{2}, \Omega=100 \mathrm{fs}^{-1}$ being a typical vibrational frequency of the physical system, and a fixed time step of $1 \mathrm{fs}$. One proper way to combine the Nosé-Hoover thermostat with a velocity Verlet integrator has been derived by Martyna and coworkers [37]. The 
adaptively biased molecular dynamics trajectories were carried out at $300 \mathrm{~K}$ with a biasing function constructed in the bidimensional range $0<\xi<300 \AA^{2}$ for bradykinin, $0<\xi<$ $600 \AA^{2}$ for the M2TMP variant, and $1000<\xi<3000 \AA^{2}$ for $\alpha$-lactalbumin, and with $0<A<1$ for the three systems. These ranges are broad enough to cover any realistic molecular conformation. Discretization was taken as 100 bins in $\xi$ and 50 bins in $A$. The width kernels were chosen as $\Delta \xi=6 \AA^{2}$ for bradykinin, $12 \AA^{2}$ for the M2TMP variant and $80 \AA^{2}$ for $\alpha$ lactalbumin, with $\Delta A=0.02$ for all systems. The flooding time was taken as $\tau_{\mathrm{F}}=0.5 \mathrm{ps}$ and $N_{W}=10$ independent walkers were propagated simultaneously, with the replication/deletion procedure applied every picosecond during the equilibration stage of $1 \mathrm{~ns}$. The simulations were then propagated for an additional 5 ns during which configurations were periodically recorded every $10 \mathrm{ps}$ for each walker. Due to computational limitations, the simulation time was shortened to $1 \mathrm{~ns}$ for $\alpha$-lactalbumin.

The replica-exchange simulations considered $N_{T}=32$ replicas in the temperature range $200-1000 \mathrm{~K}$ and a period for attempting exchanges of $\tau_{\mathrm{ex}}=1 \mathrm{ps}$. The trajectories were again propagated during $1 \mathrm{~ns}$ for equilibration, and an additional $5 \mathrm{~ns}$ to accumulate sample configurations. Note that this number of replicas makes the REMD simulations three times more demanding than the ABMD simulations.

\section{Sampling Efficiency of Adaptively Biased Molecular Dynamics}

As with many other flat-histogram schemes, the purpose of the ABMD method is to calculate free energies along reaction coordinates, biasing the sampling to enhance barrier crossing. Considering that the biomolecular force field may lack accuracy, we do not seek to characterize relative free energies here. Instead we use the method to explore broad regions of conformational space, comparing it to the more conventional replica-exchange strategy.

Efficiency will be assessed on two key features. By design, both methods are aimed at facilitating barrier crossing, and could thus sample very distant regions of the energy landscape. We will first consider the propensity of each method to locate low-energy structures, and then to contribute to solving the global optimization problem by comparing the energies of the local minima obtained from periodic quenching of the pool of configurations. In replicaexchange MD, the lowest-energy minima are expected to be explored by the low-temperature replicas, whereas at high temperatures (up to $1000 \mathrm{~K}$, which is above the folding temperature) many highly disordered conformations will be sampled. In contrast, using the ABMD method, a single trajectory should be able to sample both the low- and highenergy regions, and spend most time crossing back and forth between them. In the frame of the present work, the second comparison criterion will be the ability of the two methods to sample a large range of collision cross-sections. All distributions presented in the following were calculated based on samples of 10,000 configurations.

We have represented, in Figure 1a, the distributions of inherent structure energies obtained for triply-protonated bradykinin using the ABMD and REMD methods. Replicaexchange MD produces a roughly Gaussian distribution extended over $30 \mathrm{kcal} / \mathrm{mol}$, and a globular lowest-energy structure. Adaptively biased MD, on the other hand, leads to a much wider distribution covering $50 \mathrm{kcal} / \mathrm{mol}$, with broader shoulders on both sides, and a significant shift to higher energies relative to the REMD distribution. The most stable minimum is also globular, differs by some side chain orientation, and is higher by $1.3 \mathrm{kcal} / \mathrm{mol}$ with respect to the putative global minimum identified by quenched REMD.

The corresponding distributions for the M2TMP variant are shown in Figure 2a. Again, the REMD method yields a peaked distribution essentially centered at low energies, and with a width of $70 \mathrm{kcal} / \mathrm{mol}$. The most stable configuration has an $\alpha$-helical backbone at its center and makes two turns
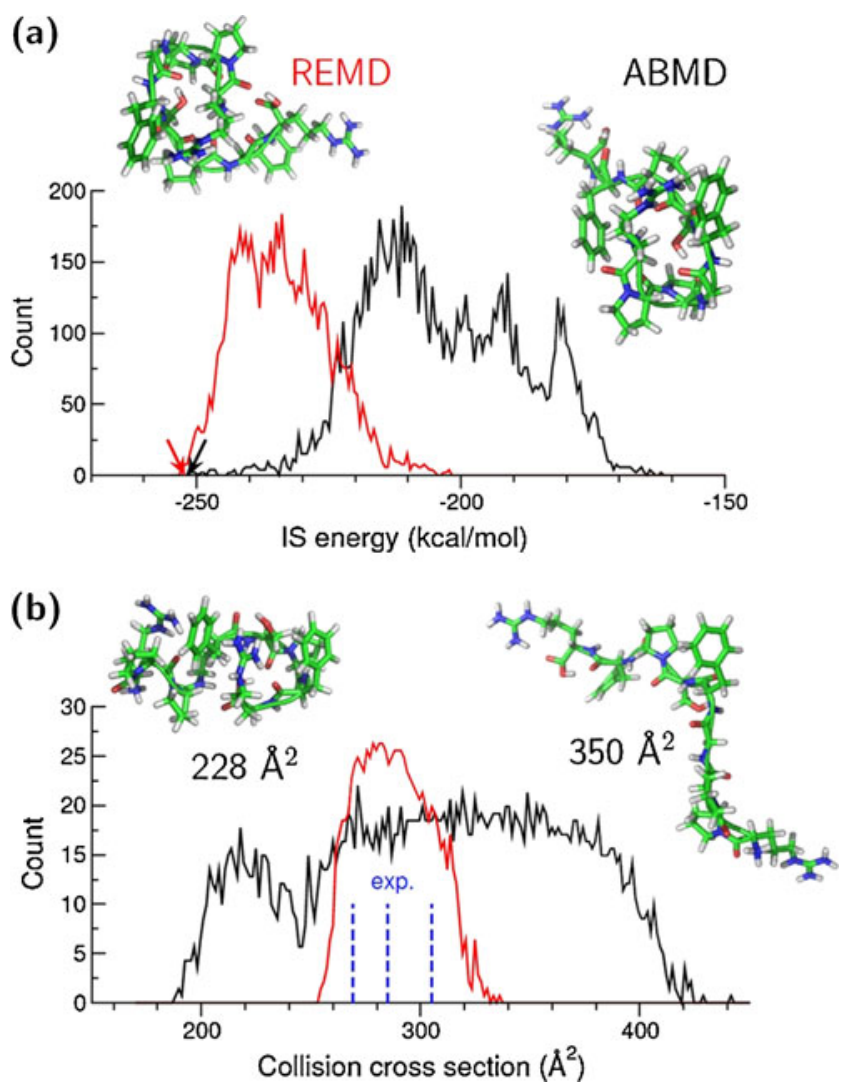

Figure 1. Statistical distributions obtained for triply protonated bradykinin from REMD (red lines) and ABMD (black lines) simulations. (a) Inherent structure (IS) energies resulting from systematic quenching. The arrows on the left sides locate the lowest-energy conformations, also depicted on the upper part of the figure. (b) Collision cross sections, with two representative $A B M D$ conformations away from the REMD distribution. The cross sections experimentally measured by Pierson et al. [27] are highlighted by vertical blue dashed lines 

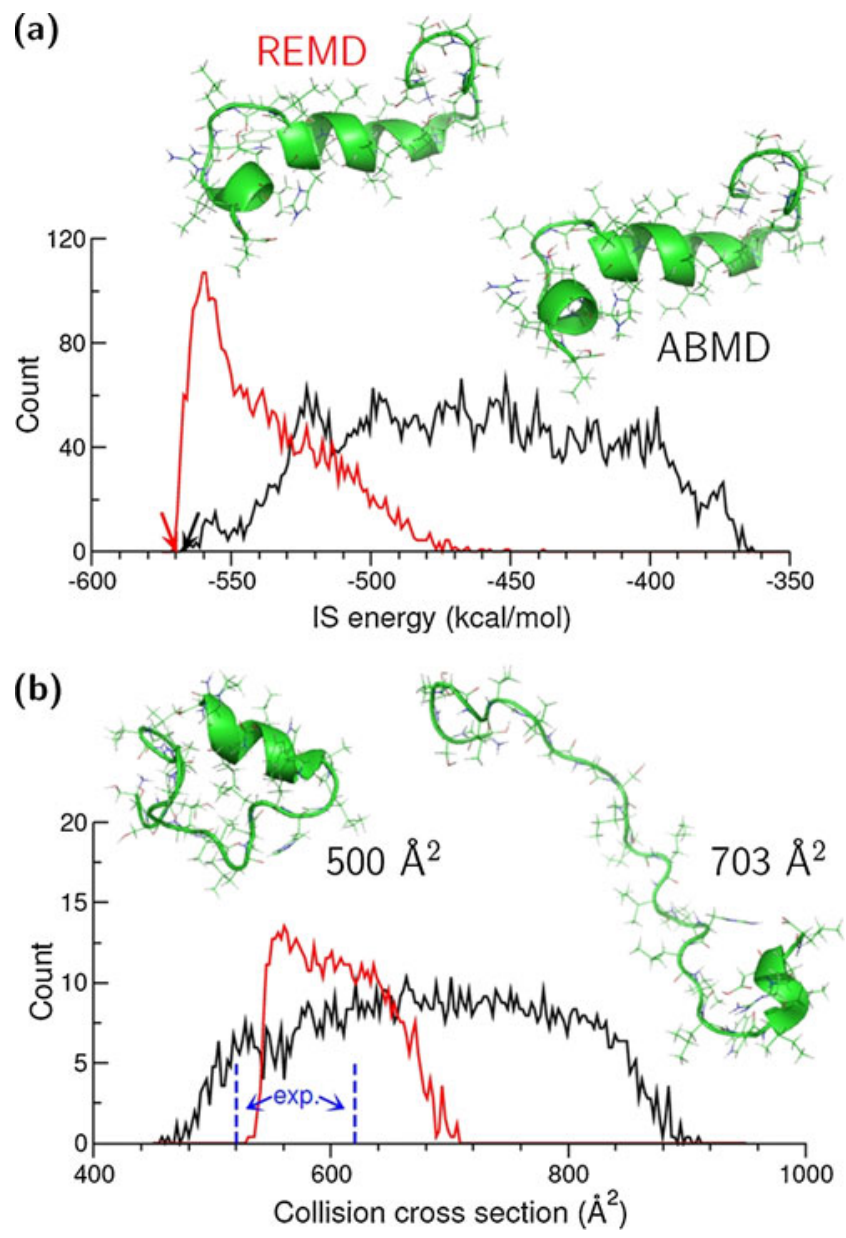

Figure 2. Statistical distributions obtained for the triply protonated G13L variant of the M2TMP peptide from REMD (red lines) and ABMD (black lines) simulations. (a) Inherent structure (IS) energies resulting from systematic quenching. The arrows on the left sides locate the lowest-energy conformations, also depicted on the upper part of the figure. (b) Collision cross sections, with two representative ABMD conformations away from the REMD distribution. The cross sections experimentally measured by Albrieux et al. [29] are highlighted by vertical blue dashed lines

at both ends. It is slightly lower in energy than the putative global minima identified in our previous paper [29], although it is very similar in shape. The distribution of inherent structures obtained with the ABMD method is much broader (about $150 \mathrm{kcal} / \mathrm{mol}$ ) and flatter. The lowest-energy inherent structure looks very similar to the REMD minimum, but has a slightly extended helical region and some minor deviations in the backbone angles near the tryptophan residue. It is also higher in energy by about $3.3 \mathrm{kcal} / \mathrm{mol}$.

These results suggest that both sampling methods are efficient for producing low-energy structures, but with some differences. For pure optimization purposes, the REMD approach is generally superior because most time is spent near the physically relevant regions (minima) whereas the adaptively biased approach wanders in the less favorable, high-energy parts of the landscape.
The distributions of collision cross-sections $\Omega$ obtained for the two peptides are represented in Figures $1 \mathrm{~b}$ and $2 \mathrm{~b}$, with the experimental values being highlighted. In the case of bradykinin, the absence of any biasing strategy in replicaexchange MD leads to a rather narrow distribution centered near $290 \AA^{2}$ and a width of $30 \mathrm{~A}^{2}$. However, as expected the adaptively biased MD method covers much more compact configurations $\left(200 \AA^{2}\right)$ and extended configurations $\left(400 \AA^{2}\right)$ alike. For this peptide, both methods are able to produce conformations that are compatible with experimental data. In the case of the M2TMP variant, the ABMD method also samples a much wider distribution of collision cross-sections and manages to locate more compact conformations than replica-exchange MD, with a higher helical content. At $1000 \mathrm{~K}$, the conformations explored by REMD are already quite extended and $\Omega$ can exceed $700 \AA^{2}$. Of the two experimentally measured collision cross-sections, only the highest value falls in the range covered by REMD. In this case, the more compact conformations not found with the force field are effectively sampled by adaptive biasing, without having to specify umbrella potentials with target values for the gyration radius.

Interestingly, for the two systems the ABMD distributions are mostly flat, even though the biasing did not act on $\Omega$ but on the $(\xi, A)$ couple. This confirms that the gyration tensor parameters provide suitable guiding coordinates for exploring a wide range of the collision cross-section.

\section{Shape Correlations}

The success of the adaptively biased MD method to generate large sets of molecular conformations covering a broad range of collision cross-sections will now be exploited more systematically in order to determine whether $\Omega$ can be approximated by other geometrical quantities that are simpler to evaluate. In their recent projection superposition approximation method [23], Bleiholder and coworkers have introduced a shape correcting term taking the concavity of the analyte into account. Following similar lines, and besides $\xi, A$ and $P$, we have also considered the surface area $s$ and the volume $v$ of the convex hull as two additional geometrical parameters containing additional information not necessarily captured by the global parameters $\xi$ and $A$ only. In addition, the possible correlation between $\Omega$ and the inherent structure energy $E_{\min }$ was also investigated. Rather than scatter plots, the data were organized into bidimensional density histograms for better clarity.

We show in Figure 3 the correlation plots between the collision cross-section and the four parameters $\xi, A, E_{\min }$ and $s$, as obtained for triply-protonated bradykinin from the ABMD trajectories. The corresponding data-clouds covered by the REMD configurations fall into subsets of points with much smaller contours. The amount of correlation between the variables can be visualized directly on the bidimensional histograms, and quantified using partial rank correlation coefficients (PRCCs), which are particularly sensitive for 

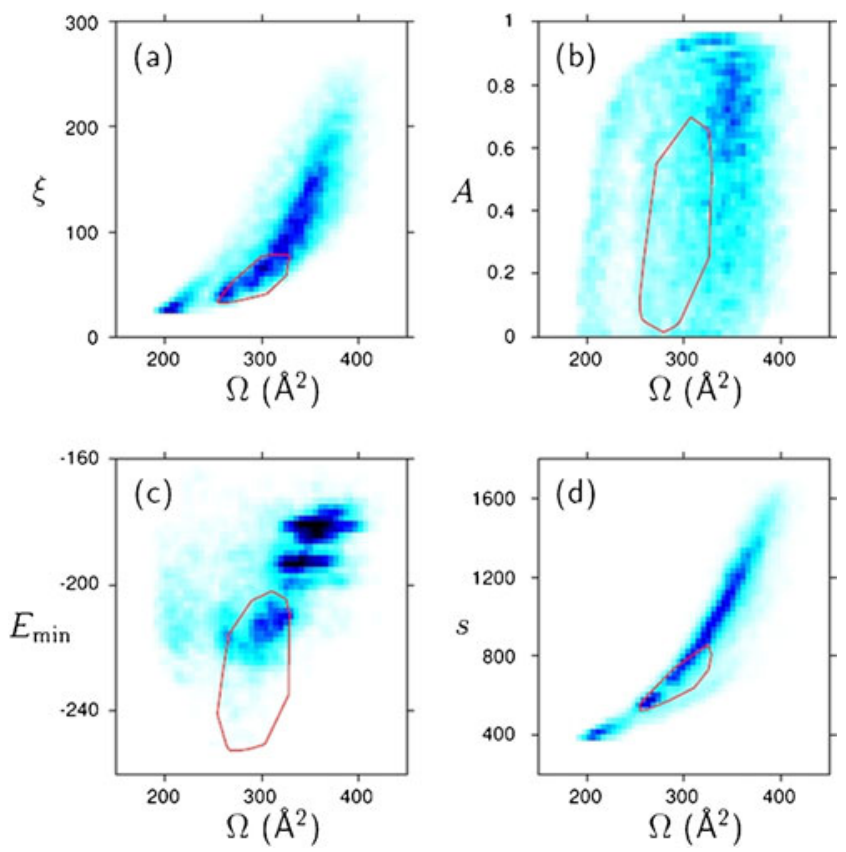

Figure 3. Correlation between the collision cross section $\Omega$ and various geometrical or energetic quantities, as obtained for triply protonated bradykinin from adaptively biased MD trajectories. The domains sampled by replica-exchange MD are located within the red polygons. (a) Square gyration radius; (b) asphericity parameter; (c) inherent structure energy; (d) surface area of the convex hull

monotonic, yet highly nonlinear data [38]. From these plots, and as already pointed out in our previous article [24], the square gyration radius correlates reasonably well with $\Omega$, and shows at least a monotonic behavior. The asphericity parameter, on the other hand, does not display any particular correlation, except for unfolded structures that are mostly elongated and lie in the upper right part of the $(\Omega, A)$ diagram. The prolateness parameter $P$ does not provide a much higher degree of correlation. Neither correlation is found between the inherent structure energy and the collision cross-section for this peptide. Even though the least stable conformations are, expectedly, the most extended, there is no evidence that the most stable structure is the most compact. The highest degree of correspondence between $\Omega$ and a single geometric quantity is reached with the surface and volume of the convex hull, as seen in Figure $5 \mathrm{~d}$ for the case of $s$, the $(\Omega, v)$ diagram being very similar. The PRCCs calculated for the bradykinin data, reported in Table 1, confirm the trends observed on the histograms, which suggests that correlations are maximum with $\xi$ and $s$.

Figure 6 shows the corresponding histograms obtained for the M2TMP variant, with their PRCCs indices also given in Table 1. The same general conclusions can be drawn as for the smaller peptide, the degree of correlation with $\Omega$ being even higher in the case of the surface area.

Although these geometric parameters are highly correlated to the collision cross-section, visual inspection of Figures 3 and 4 indicates that their relation is clearly non linear. An optimal index $\chi$ combining other geometrical quantities can be determined by maximizing the statistical correlation with $\Omega$. Variables presenting systematically low correlation with $\Omega$, such as $A$ or $P$, can be safely ignored, and the best performance was found for the subset $(\xi, s, v)$. Using $E_{\text {min }}$ did not improve the representation. For the two systems considered, we eventually found a satisfactory model, based on a polynomial relation between the logarithms:

$$
\begin{aligned}
\log \chi= & a_{0}+a_{\xi} \log \xi+a_{s} \log s+a_{v} \log v \\
& +a_{\xi \mathrm{s}}(\log \xi) \times(\log s)+a_{\xi \mathrm{v}}(\log \xi) \times(\log v)+a_{s v}(\log s) \times(\log v) \\
& +a_{\xi s v}(\log \xi) \times(\log s) \times(\log v)
\end{aligned}
$$

where the eight parameters $a_{0}, a_{\xi}, \ldots, a_{\xi v}$ were adjusted to maximize the correlation between $\Omega$ and $\chi$ through a linear least-squares procedure. The mathematical template of Equation (7) does not have a clear physical background, because even the dimensionalities of the combined parameters are not preserved when taking the logarithms. However, this form for representing the collision crosssection is surprisingly effective, at least for the two peptides studied here. For these systems, the final values of the parameters are explicitly shown in Table 2, and the correlation is visualized in Figure 5. Undoubtly, the phenomenologic parameter $\chi$ quantitatively mimics the variations of $\Omega$, the standard relative uncertainty being about $3 \%$ for both peptides. The present results thus show that the collision cross-section can be very well captured by a limited number of purely geometrical quantities. Moreover, the expansion coefficients for both molecules are remarkably similar, which might indicate that some of them could be constrained a priori.

We have questioned the validity of this geometrical order parameter by performing ABMD simulations on the much larger $\alpha$-lactalbumin protein, starting from the PDB structure (ID: $1 \mathrm{~F} 6 \mathrm{~S}$ ) from which the calcium cation, the few water

Table 1. Partial Rank Correlation Coefficients Between the Collision Cross Section $\Omega$ and the Initial Set of Geometrical and Energetic Properties, Obtained

\begin{tabular}{|c|c|c|c|c|c|c|}
\hline Peptide & $\xi$ & $A$ & $P$ & $E_{\min }$ & $s$ & $v$ \\
\hline Bradykinin & $0.55(4)$ & $0.01(5)$ & $-0.05(5)$ & $0.12(6)$ & $0.24(6)$ & $-0.05(6)$ \\
\hline M2TMP & $0.16(5)$ & $-0.02(5)$ & $0.02(5)$ & $0.05(5)$ & $0.38(5)$ & $-0.20(6)$ \\
\hline
\end{tabular}
for Triply-Protonated Bradykinin and the G13L Variant of M2TMP from Sets of 10,000 Configurations Sampled by Adaptively Biased Molecular Dynamics Simulations 

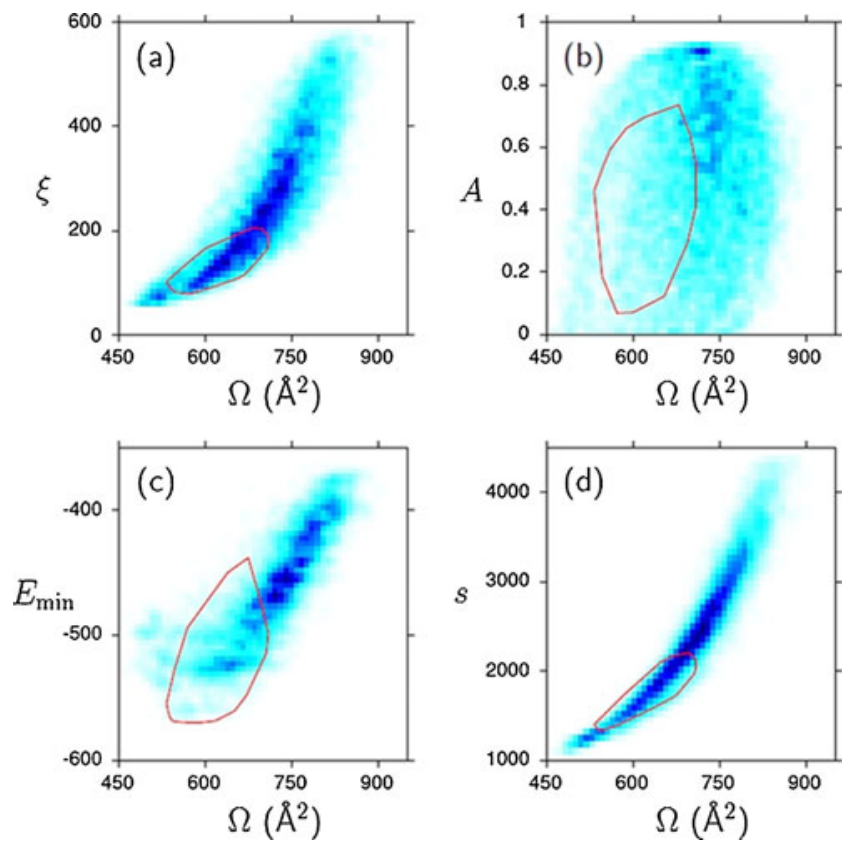

Figure 4. Correlation between the collision cross section $\Omega$ and various geometrical or energetic quantities, as obtained for the G13L variant of M2TMP from adaptively biased MD trajectories. The domains sampled by replica-exchange MD are located within the red polygons. (a) Square gyration radius; (b) asphericity parameter; (c) inherent structure energy; (d) surface area of the convex hull

molecules, and the disulfide bridges were removed for added flexibility. Moreover, all the acidic residues were deprotonated in order to reach a total charge of -8 . From a sample of 3000 configurations, the correlation between the gyration radius, the surface and volume of the convex hull and the collision cross-section was identified using the same template of Equation (7). The parameters of this adjustment, given in Table 2, are remarkably close to those of the two smaller systems, suggesting that the model could possibly be generalized for a broader class of biomolecules. In the correlation plot, shown in Figure 6, two particular lowenergy conformations have been depicted. The crystallographic structure, used at the beginning of the simulation, is among the most compact conformations with a collision cross-section close to $1540 \AA^{2}$. Systematic quenching of the conformations sampled during the simulations yields more stable minima, the putative global minimum being lower in energy by more than $300 \mathrm{kcal} / \mathrm{mol}$ and exhibiting a rather
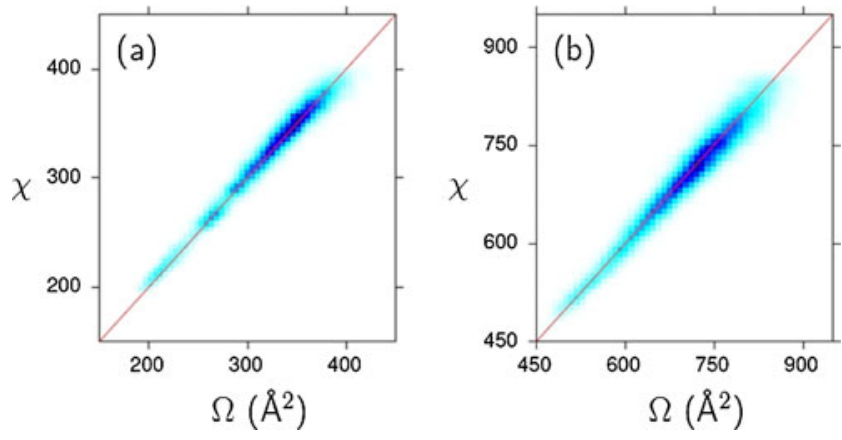

Figure 5. Maximum correlation obtained between the collision cross section $\Omega$ and a purely geometrical index $x$ defined according to Equation (7), for (a) triply protonated bradykinin; (b) the G13L variant of M2TMP. The red lines locate the diagonal $X=\Omega$ corresponding to optimal correlation

extended character without significant helical or strand secondary structures, and with $\Omega=1820 \AA^{2}$. Ion mobility experiments are currently under way in our laboratory in order to confirm the predicted difference in stability between the crystallographic and gas-phase conformations.

This third example further supports the high correlation between the collision cross-section and purely geometric observables. Of course, even more examples would be needed for a robust generalization of this model. However, the possibility to represent the collision cross-section by straightforward geometrical observables could be exploited to design much faster algorithms for estimating $\Omega$ in conformational samples of large molecular systems, without having to repeat the tedious calculation of $\Omega$ for each structure. A small subset of conformations, generated by hand or by a short, high-temperature molecular simulation could for instance be used only to estimate the fitting parameters for $\chi$, which would be subsequently used in place of $\Omega$. Having a better representation of $\Omega$ would greatly help the sampling as well. Because all components of $\chi$ are differentiable, this parameter can be used as the single guiding parameter, either in adaptively biased MD or in umbrella sampling targeting specific experimental structures. A straightforward use of this correlation would be to process large samples of structures generated by (unbiased) conventional simulations, and for which the individual calculation of $\Omega$ could thus be avoided.

It is also remarkable that the optimal parameters representing $\Omega$ show a rather weak dependence on the system, which suggests that the template of Equation (7)

Table 2. Best-fit Parameters $a_{0}, \ldots, a_{\xi s v}$ of the Phenomenological Function $\chi$ that Maximizes Correlation with the Collision Cross Section [Equation 7], as Obtained for Triply-Protonated Bradykinin, the G13L Variant of M2TMP, and the $\alpha$-Lactalbumin Protein from Sets of Configurations Sampled by Adaptively Biased Molecular Dynamics Simulations

\begin{tabular}{lcccccccc}
\hline Peptide & $\alpha_{0}$ & $\alpha_{\xi}$ & $\alpha_{s}$ & $\alpha_{v}$ & $\alpha_{\xi s}$ & $\alpha_{\xi v}$ & $\alpha_{s v}$ & $\alpha_{\xi s v}$ \\
\hline Bradykinin & -11.154 & 3.385 & 1.820 & 2.247 & -0.354 & -0.498 & -0.247 \\
M2TMP & -15.855 & 3.321 & 2.152 & 2.545 & -0.276 & -0.446 & -0.247 & 0.055 \\
$\alpha$-lactalbumin & -18.452 & 2.940 & 2.449 & 2.264 & -0.287 & -0.282 & -0.210 & 0.028 \\
\hline
\end{tabular}




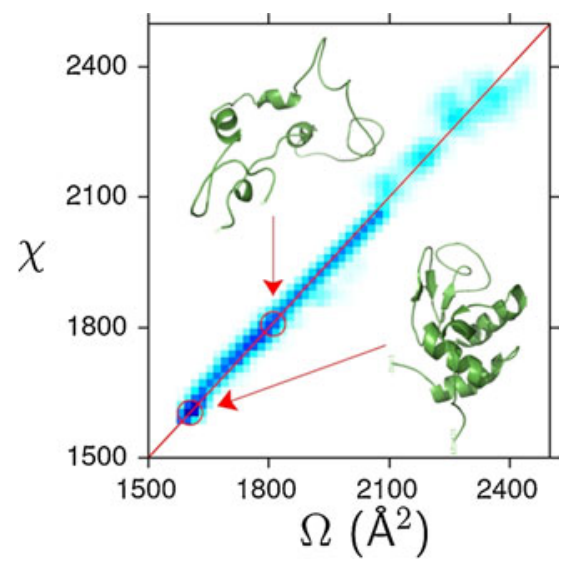

Figure 6. Maximum correlation obtained between the collision cross section $\Omega$ and a purely geometrical index $\mathrm{X}$ defined according to Equation (7), for the a-lactalbumin protein. The red line locate the diagonal $X=\Omega$ corresponding to optimal correlation

may be valid for a broader class of biomolecules. More robust values could be obtained by performing a global adjustment on a series of compounds, but this would require additional input from different systems. Also, we notice that the three properties contained in $\chi$ have different scalings with the number of atoms, $\xi$ and $s$ behaving as $N^{2}$ while $v$ scales as $N^{3}$. These dependencies on size could be explicitly shown in the expression of $\chi$, and systematic exploration of polymers with various lengths could be used to derive new unscaled parameters. Of course, the specific values of those parameters will depend in practice on the numerical details needed to estimate the collision cross-section, such as the temperature or the atomic mass of the diffusing gas, but the limiting factor remains the evaluation of $\Omega$.

\section{Conclusions}

Computational modeling can be of great assistance in interpreting ion mobility measurements, as it can locate molecular conformations that are non-trivial, chemically realistic, and highly stable at the same time. From the theoretical point of view, different answers may be provided by different descriptions of the interactions because the intrinsic stability of a molecule depends on a number of factors, including temperature and environment. In the present work, we have presented a computational scheme based on adaptively biased molecular dynamics for producing large sets of conformations that cover broad ranges of collision cross-section, and from which structures compatible with IMS data could be retrieved. Our implementation employed the two lowest moments of the gyration tensor to guide the sampling and achieve flat-histogram sampling in both the square gyration radius and the asphericity parameters, but only the former was found to have a significant effect on the collision cross section. In retrospect, it is thus probably sufficient to bias on the gyration radius to ensure a wide sampling in the collision cross section is achieved. When applied to representative 9- and 25-residue peptides, the adaptive method happens to be clearly more powerful than replica-exchange MD in exploring wide ranges of cross sections, in the two opposite directions of more compact and more extended structures. The ABMD approach is also computationally less expensive because fewer replicas are needed. However, owing to its greater proportion of time spent near barriers, it is not as efficient for solving the global optimization problem.

The large conformational samples gathered by the method were statistically analyzed in terms of the possible correlation between the collision cross section and various geometrical and energetic properties. Although the square gyration radius generally correlates well, an even higher correlation was obtained with the surface and volume of the convex hull. This suggested a phenomenologic way of representing the collision cross section by combining purely geometric quantities with numerical parameters determined by maximizing the overall sensitivity. Although not based on physical grounds, the resulting expression seems generally applicable to peptides and even to proteins. So far, our results indicate that the numerical parameters should be adjusted to each new system, even though they seem to lie in well-defined ranges for the present class of biomolecules. It would be useful to search for more universal empirical relations, possibly involving additional quantities such as the higher moments of the gyration tensor. Determining the parameters appropriate for a new analyte should not pose a significant bottleneck, as this would require only a few very different conformations. The finding of a purely geometric and explicit, hence, computationally very inexpensive, way of capturing the collision cross section for very different conformations should greatly facilitate the systematic evaluation of collision cross-sections for large systems, especially in concern with the need to relate experiments not only to static values but also to statistical distributions [24, 29]. From the computational point of view, this also paves the way to design more efficient exploration algorithms for interpreting IMS data using biased molecular dynamics simulations.

Having a powerful sampling method with a computationally inexpensive substitute for $\Omega$ also opens great perspectives for the interpretation of experiments combining ion mobility to other techniques [39-44]. This task is particularly challenging for optical spectroscopy, the analysis of which requires comparison with high-level, time-consuming calculations. In this case, an efficient computational strategy consists of scanning molecular conformations based on their collision cross-sections before further analysis [42]. Such a procedure can be applied with a restraint potential on $\chi$ (rather than $\Omega$ ) acting as an IMS filter to perform a conformational sampling resolved in ion mobility.

\section{References}

1. Jarrold, M.F.: Unfolding, Refolding and Hydration of Proteins in the Gas Phase. Acc. Chem. Res. 32, 360-367 (1999) 
2. Barran, P.E.; Polfer, N.C.; Campopiano, D.J.; Clarke, D.J.; LangridgeSmith, P.R.R.; Langley, R.J.; Govan, J.R.W.; Maxwell, A.; Dorin, J.R.; Millar, R.P.; Bowers, M.T.: Is it biologically relevant to measure the structures of small peptides in the gas-phase? Int. J. Mass Spectrom. 240, 273-284 (2005)

3. Bohrer, B.C.; Merenbloom, S.I.; Koeniger, S.L.; Hilderbrand, A.E.; Clemmer, D.E.: Biomolecule Analysis by Ion Mobility Spectrometry. Annu. Rev. Anal. Chem., 1, 293-327 (2008)

4. Politis, A., Park, A.Y., Hyung, S.-J., Barsky, D., Ruotolo, B.T.; Robinson, C.V.: Integrating Ion Mobility Mass Spectrometry with Molecular Modelling to Determine the Architecture of Multiprotein Complexes. PLOS ONE 5, e12080 (2010)

5. Canon, F.; Ballivian, R.; Chirot, F.; Antoine, R.; Sarni-Manchado, P.; Lemoine, J.; Dugourd, P. Folding of a Salivary Intrinsically Disordered Protein upon Binding to Tannins. J. Am. Chem. Soc. 133, 7847-7852 (2011)

6. Bernstein, S. L.; Dupuis, N. F.; Lazo, N. D.; Wyttenbach, T.; Condron, M. M., Bitan, G.; Teplow, D. B.; Shea, J.-E.; Ruotolo, B. T.; Robinson, C. V.; Bowers, M. T., Amyloid- $\beta$ protein oligomerization and the importance of tetramers and dodecamers in the aetiology of Alzheimer's disease. Nat. Chem. 1, 326-331 (2009)

7. Uetrecht, C.; Barbu, I. M.; Shoemaker, G. K.; van Duijn, E.; Heck, A. J. R. Interrogating viral capsid assembly with ion mobility-mass spectrometry. Nat. Chem. 3, 126-132 (2011)

8. Chen, L.; Gao, Y. Q.; Russell, D. H. How alkali metal ion binding alters the conformer preferences of gramicidin A: A molecular dynamics and ion mobility study. J. Phys. Chem. A 116, 689-696 (2012)

9. Bleiholder, C.; Suhai, S.; Paizs, B. Revising the proton affinity scale of the naturally occurring $\alpha$-amino acids. J. Am. Soc. Mass Spectrom., 17, 1275-1281 (2006)

10. Rodriquez, C. F.; Orlova, G.; Guo, Y.; Li, X.; Siu, C.-K.; Hopkinson, A. C.; Siu, K. W. M. Gaseous Bradykinin and Its Singly, Doubly, and Triply Protonated Forms: A First-Principles Study. J. Phys. Chem. B, 110, 7528-7537 (2011)

11. Strittmatter, E. F.; Williams, E. R. Structures of Protonated Arginine Dimer and Bradykinin Investigated by Density Functional Theory: Further Support for Stable Gas-Phase Salt Bridges. J. Phys. Chem. A, 104, 6069-6076 (2011)

12. Sugita, Y.; Okamoto, Y. Replica-exchange molecular dynamics method for protein folding. Chem. Phys. Lett. 314, 141-151 (1999)

13. Kumar, S.; Rosenberg, J. M.; Bouzida, D.; Swendsen, R. H.; Kollman, P. A. The weighted histogram analysis method for free-energy calculations on biomolecules. I. The method. J. Comput. Chem., 13, 1011-1021. (1992)

14. Wang, F.; Landau, D. P. Efficient, Multiple-Range Random Walk Algorithm to Calculate the Density of States. Phys. Rev. Lett., 86, 2050-2053 (2001)

15. Poulain, P.; Calvo, F.; Antoine, R.; Broyer, M.; Dugourd, Ph., Performances of Wang-Landau algorithms for continuous systems. Phys. Rev. E 73, 056704(1-11) (2006)

16. Marsili, S.; Barducci, A.; Chelli, R.; Procacci, P.; Schettino, V. Selfhealing Umbrella Sampling: a non equilibrium approach for quantitative free energy calculations. J. Phys. Chem. B, 110, 14011-14013 (2006)

17. Chen, L.; Shao, Q.; Gao, Y.-Q.; Russell, D. H. Molecular dynamics and ion mobility study of model $\beta$-hairpin peptide, Trpzip 1. J. Phys. Chem. A 115, 4427-4435 (2011)

18. Laio, A.; Parrinello, M. Escaping free-energy minima. Proc. Natl. Acad. Sci. U. S. A., 99, $12562-12566$ (2002)

19. von Helden, G. Hsu, M. T. Gotts, N.; Bowers, M. T. Carbon cluster cations with up to 84 atoms: structures, formation mechanism, and reactivity. J. Phys. Chem., 97, 8182-8192 (1993)

20. Shvartsburg, A. A.; Jarrold, M. F. An exact hard-spheres scattering model for the mobilities of polyatomic ions. Chem. Phys. Lett., 261, 8691 (1996)

21. Wyttenbach, T.; von Helden, G.; Batka, J. J.; Carlat, D.; Bowers, M. T. Effect of the Long-range potential on ion mobility measurements. $J$. Am. Soc. Mass Spectrom. 8, 275-282 (1997)

22. Mesleh, M. F., Hunter, J. M., Shvartsburg, A. A., Schatz, G. C.; Jarrold, M. F. Structural Information from Ion Mobility Measurements: Effects of the Long-Range Potential. J. Phys. Chem. 100, 16082-16086 (1996)
23. Bleiholder, C.; Wyttenbach, T.; Bowers, M. T. A novel projection approximation algorithm for the fast and accurate computation of molecular collision cross sections (I). Method. Int. J. Mass Spectrom. 308, 1-10 (2011)

24. Chirot, F.; Calvo, F.; Albrieux, F.; Lemoine, J.; Tsybin, Y. O.; Dugourd, P. Statistical Analysis of Ion Mobility Spectrometry. I. Unbiased and guided replica-exchange molecular dynamics. J. Am. Soc. Mass Spectrom., 23, 386-396 (2012)

25. Calvo, F. Sampling along reaction coordinates with the Wang-Landau method. Mol. Phys. 100, 3421-3427 (2002)

26. Babin, V.; Roland, C.; Sagui, C. Adaptively biased molecular dynamics for free energy calculations. J. Chem. Phys. 128, 134101 (2008)

27. Pierson, N. A.; Valentine, S. J.; Clemmer, D. E. Evidence for a QuasiEquilibrium Distribution of States for Bradykinin $[\mathrm{M}+3 \mathrm{H}]^{3+}$ Ions in the Gas Phase. J. Phys. Chem. B, 114, 7777-7783 (2010)

28. Pierson, N. A.; Chen, L.; Valentine, S. J.; Russell, D. H.; Clemmer, D. E. Number of Solution states of bradykinin from ion mobility and mass spectrometry measurements. J. Am. Chem. Soc. 133, 13810-13813 (2011)

29. Albrieux, F.; Ben Hamidane, H.; Calvo, F.; Chirot, F.; Tsybin, Y. O.; Antoine, R.; Lemoine, J.; Dugourd, P. Structural Preferences of GasPhase M2TMP Monomers upon Sequence Variations. J. Phys. Chem. A 115, 4711-4718 (2011)

30. Wang, J. Cieplak, P.; Kollman, P. How well does a restrained electrostatic potential (RESP) model perform in calculating conformational energies of organic and biological molecules? J. Comput. Chem. 21, 1074, 1049 (2000)

31. Swendsen, R. H.; Wang, J.-S. Replica Monte Carlo Simulation of SpinGlasses. Phys. Rev. Lett. 57, 2607-2609 (1986)

32. Torrie, G. M.; Valleau, J. P. Nonphysical sampling distributions in Monte Carlo free-energy estimation: Umbrella sampling. J. Comput. Phys. 23, 187-199. (1977)

33. Raiteri, P.; Laio, A.; Gervasio, F. L.; Micheletti, C.; Parrinello, M. Efficient Reconstruction of Complex Free Energy Landscapes by Multiple Walkers Metadynamics. J. Phys. Chem. B, 110, 3533-3539 (2006)

34. Calvo, F. Free-energy landscapes from adaptively biased methods: Application to quantum systems. Phys. Rev. E, 82, 046703 (2010)

35. Wei, G.; Eighinger, B. E. On shape asymmetry of Gaussian molecules. J. Chem. Phys., 93, 1430-1435 (1990)

36. Barber, C. B.; Dobkin, D. P.; Huhdanpaa, H. The quickhull algorithm for convex hulls. ACM Trans. Math. Software 22, 469-483 (1996)

37. Martyna, G. J.; Tobias, D. J.; Klein, M. L. Constant pressure molecular dynamics algorithms. J. Chem. Phys. 101, 4177-4189 (1994)

38. Hamby, D. M. A review of techniques for parameter sensitivity analysis of environmental models. Environmental Monitoring and Assessment, 32, 135-154 (1994)

39. Fromherz, R. Ganteför, G.; Shvartsburg, A. A. Isomer-Resolved Ion Spectroscopy. Phys. Rev. Lett. 89, 083001 (2002)

40. Polfer, N. C. Bohrer, B. C. Plasencia, M. D. Paizs, B.; Clemmer, D. E. On the dynamics of fragment isomerization in collision-induced dissociation of peptides. J. Phys. Chem. A, 112, 1286-1293 (2008)

41. Misaizu, F., Hori, N., Tanaka, H., Komatsu, K., Furuya, A.; Ohno, K. Isomer-selected photoreactions of gas-phase cluster ions. Eur. Phys. J. $D$, 52, 59-62 (2009)

42. Poully, J. C.; Lecomte, F.; Nieuwjaer, N.; Manil, B.; Schermann, J. P.; Desfrancois, C.; Gregoire, G.; Ballivian, R.; Chirot, F.; Lemoine, J.; Calvo, F.; Antoine, R.; Dugourd, P. Combining ion mobility mass spectrometry and infrared multiphoton dissociation spectroscopy to probe the structure of gas-phase vancomycin-Ac2LKDADA noncovalent complex. Int. J. Mass Spectrom., 297, 28-35 (2010)

43. Zucker, S.; Lee, S.; Webber, N.; Valentine, S.; Reilly, J.; Clemmer, D. An Ion Mobility/Ion Trap/Photodissociation Instrument for Characterization of Ion Structure. J. Am. Soc. Mass Spectrom., 22, 1477-1485 (2011)

44. Joly, L.; Antoine, R.; Albrieux, F.; Ballivian, R.; Broyer, M.; Chirot, F.; Lemoine, J.; Dugourd, P.; Greco, C.; Mitrić, R.; Bonačić-Koutecký, V. Optical and Structural Properties of Copper-Oxytocin Dications in the Gas Phase. J. Phys. Chem. B, 113, 11293-11300 (2009) 